\title{
Altered search speed and growth: social versus independent foraging in two pelagic juvenile fishes
}

\author{
Clifford H. Ryer*, Bori L. Olla
}

\author{
Alaska Fisheries Science Center, National Marine Fisheries Service, Hatfield Marine Science Center, \\ Newport, Oregon 97365, USA
}

\begin{abstract}
Prior studies have demonstrated that juvenile walleye pollock Theragra chalcogramma forage socially in schools for spatially and temporally clumped food, but forage more independently for spatially and temporally dispersed food. One advantage of social foraging is that fish in schools may be able to locate more food clumps than fish foraging individually. However, data also indicate that walleye pollock swim faster when foraging socially. We conducted laboratory experiments to evaluate the effect of food distribution upon the energetic foraging costs incurred by juvenule walleye pollock and sablefish Anaplopoma fimbria. We predicted that when given identical rations, fish receiving clumped food would swim faster, expending more energy, and therefore grow more slowly than fish receiving dispersed food. After $2 \mathrm{wk}$ under these 2 foraging regımes, juvenile walleye pollock receiving clumped food swam $50 \%$ faster, but experienced $19 \%$ lower growth, than walleye pollock receiving dispersed food. Sablefish demonstrated only a weak swim speed response, with no difference in growth between food distributions. Our results demonstrate that although social foraging may increase encounter rates with food, in some species there may also be an energetic cost for this behavior, which will have an influence upon energetic efficiency, potentially affecting growth and survival.
\end{abstract}

KEY WORDS: Bioenergetıcs · Foraging strategy - Growth - Milkshake effect - Shoaling - Swimming speed

\section{INTRODUCTION}

Food distribution is an important factor influencing the types of social interaction that occur between conspecifics. Among fish, this is perhaps best recognized with regard to territorial species, where food distribution and abundance, combined with competitor density, determine the occurrence and size of feeding territories (Brown 1964, Grant 1993) Similarly, among gregarious species, food distribution can determine whether or not fish form cohesive groups or torage more independently. This is exemplified by the behavior of juvenile walleye pollock (Ryer \& Olla 1992, 1995), a pelagic gadid of the north Pacific Ocean, which supports a large commercial fishery. Juveniles less than 1 yr old are typically found at depths less than $200 \mathrm{~m}$ (Brodeur \& Wilson 1996), where they forage for

\footnotetext{
·E-mail: ryerc@ccmail.orst.edu
}

planktonic prey (Grover 1990, 1991). The distributions of these prey may vary greatly, both spatially and temporally, depending upon oceanographic conditions (Stavn 1971, Mackas \& Boyd 1979, Zeldis \& Jillett 1982, Mackas et al. 1985) and prey behavior (Hamner et al. 1983, Hamner 1984, O'Brien 1989). Laboratory studies demonstrate that when food is spatially clumped and temporally synchronous, juvenile walleye pollock forage socially (Ryer \& Olla 1992), and, when food is spatially and temporally dispersed, they forage independently (Ryer \& Olla 1995).

This type of social foraging is characterized by fish responding to food discovery behaviors elicited by other fish, resulting in congregation of fish at the location where a clump has been found, i.e. local enhancement, as well as frequent congregation whenever individuals make sudden movements that attract the attention of school-mates. As a result, individuals tend to follow one another about, engaging in loose schooling behavior (Ryer \& Olla 1995). This behavior allows 
the individuals of a school to locate food clumps more rapidly than they could as individuals (Pitcher et al. 1982) Provided clumps are either large relative to the needs of the school or are ephemeral, such that there is little competition between individuals when exploiting a clump, social foraging should result in increased individual foraging efficiency (Baird et al. 1991). In contrast, fish foraging independently do not engage in schooling (Ryer \& Olla 1995). Independent foraging should be more efficient than group foraging when food is dispersed because individuals do not waste energy responding to food discovery cues of others; a single zooplankter would likely be consumed before other fish could arrive and compete.

We have observed that juvenile walleye pollock foraging socially for clumped food swim faster than do fish foraging independently for dispersed food (unpubl. data). Since it requires greater energy expenditure to swim faster (Tytler 1969, Furnoll 1987), we surmise that the energetic cost of foraging socially for clumped food must be greater than that of foraging independently for dispersed food. This is potentially important because energy costs associated with searching for prey may be 3 or 4 times the standard metabolic rate (Ware 1978, Boisclair \& Sirois 1993), representing a large portion of respiratory metabolism and playing a significant role in determining the amount of energy available for growth. Beyond its obvious importance for interpreting and predicting the growth rates of fish in the field, knowledge of how environmental factors such as food distribution influence the occurrence and energetics of social foraging helps to elucidate the evolutionary processes that have selected for schooling behavior in fish, as well as flocking and herding behaviors in other animals.

Our observations of juvenile walleye pollock and a second species, juvenile sablefish Anaplopoma fimbria, suggest that in addition to swimming speed, the complexity of the fish's swim path, i.e. its sinuosity, may also be influenced by food distribution. Like walleye pollock, sablefish are also an important commercial species of the north Pacific (Hart 1973). Although little is known about their feeding behavior, our observations in the laboratory indicate that, like walleye pollock, juvenile sablefish school readily and utilize social cues to locate food. In preliminary studies we observed that both species appeared to swim a straighter path, with longer movements between turns and direction reversals, when searching for clumped food as opposed to dispersed food. Many animal species adopt less-sinuous search paths when foraging for clumped food (Smith 1974, Williamson 1981, Coughlin et al, 1992, Ferran et al. 1994). This type of ranging search behavior should facilitate the location of food clumps (Bond 1980). In addition, a straightening of swim paths would be expected when a fish joins a school and must coordinate its movements with those of other individuals.

In this paper we report on experiments designed to test the hypothesis that the energetic costs associated with school-based foraging for clumped food are greater than those associated with independent foraging for dispersed food. We conducted these experiments on both juvenile walleye pollock and juvenile sablefish, our prior observations being that sablefish behavior was similar enough to pollock that we could expect both species to respond similarly to the differing food distributions. For each species, we acclimated replicate groups of fish to either dispersed or clumped food and then measured the swimming speed of individuals while they were foraging and monitored their growth over 2 wk. Since groups were given identical rations, regardless of how it was distributed, we predicted that fish foraging socially for clumped food would swim faster, expending more energy, and therefore grow less than fish foraging more independently for dispersed food. We also tested the hypothesis that fish foraging socially for clumped food would swim less-sinuous search paths than fish foraging independently for dispersed food

\section{MATERIALS AND METHODS}

Experimental protocols. Juvenile walleye pollock were collected at Port Townsend, Washington, USA, and returned to the Hatfield Marine Science Center. where they were maintained in $340 \mathrm{l}$ circular seawater tanks. After 2 wk, 4 groups of 8 fish each were haphazardly drawn from these stock tanks. Fish size ranged from 61 to $76 \mathrm{~mm}$ total length. Each fish was anesthetized with MS-222, measured for total length and body weight and then given a unique fin clip to distinguish it from others in its group. Fin clips consisted of taking a small notch out of the upper or lower lobe of the caudal fin, the dorsal, the left or right pectorals, or some combination of these. These fin clips remained recognizable for the 2 wk duration of the experiment, allowing us to track the growth of individuals. Groups were then transferred to $3700 \mathrm{l}$ circular flow through seawater arenas (width $2.3 \mathrm{~m} \times$ depth $0.9 \mathrm{~m}$ ) equipped with automated food delivery systems. Food was conveyed through tubes and delivered just below the water's surface at 10 locations distributed throughout each arena (see Ryer \& Olla 1995 for detailed description) This allowed dispensing of $1.5 \mathrm{~mm}$ food pellets in either (1) a spatially dispersed and temporally asynchronous pattern, with individual pellets appearing at random intervals (hereafter dispersed food) or (2) a spatially clumped and temporally 
synchronous pattern, with clumps of 10 to 20 pellets appearing at random intervals. Two groups received dispersed food while the other 2 received clumped food. In both treatments fish received the same $3 \%$ body weight ration over a $4 \mathrm{~h}$ feeding period each day. The $4 \mathrm{~h}$ duration of the feeding period was chosen for strictly logistical reasons. We estimate that the average time between dispersed food pellets was approximately 20 to $40 \mathrm{~s}$, while the average time between clumps was 5 to $10 \mathrm{~min}$. Observations confirmed that all food was consumed during the feeding period. After $1 \mathrm{wk}$ fish were removed from the arena, reweighed and measured and then returned to their arenas. Rations for each group were recalculated and the experiment was continued for one more week before fish were removed, measured and weighed for the last time. This 2 wk sequence was repeated with another 4 groups, resulting in 4 replicate groups for each food distribution. For each group, we calculated average fish weights at the end of each week. Using these averages we then calculated percentage weight change for each group over both weeks.

Juvenile sablefish were collected with a neuston net off Newport, Oregon, transported to the laboratory and maintained in 340 l circular seawater tanks. Fish selected for the experiment ranged in size from 49 to $85 \mathrm{~mm}$ total length. Experiments with sablefish were conducted in the same manner as the experiments with walleye pollock, with the exceptions that there were 6 replicates of each food distribution and sablefish received a $6 \%$ body weight daily ration to accommodate their intrinsically greater growth potential.

Behavioral observations. Overhead video recordings were made of each group at the end of each week during their normal feeding period. For 4 of the pollock groups, 2 each of clumped and dispersed treatments, videotapes from the first week were accidently destroyed, leaving tapes for only 2 replicates for each food distribution from the first week. From the remaining video tapes, 3 behavioral measures were quantified: congregation frequency, swim speed, and swim path sinuosity. A congregation was defined as having occurred when all 8 fish were within a circular area, as viewed from above, with a diameter two-fifths that of the arena, i.e. $16 \%$ of arena area. Preliminary observations indicated that such congregations were common among clumped food foragers, but less frequent among dispersed food foragers. Congregations were counted during each of four 10 min periods beginning at 0,60 , 120 and 160 min during the feeding period. In all cases, congregations resulted from fish altering their direction of movement or speed to converge in one area of the tank. This was followed by several seconds of milling behavior before the group either partially or completely dispersed. We did not count congregations where fish were directly responding to a food introduction event. Swim speed and swim path sinuosity were measured by digitizing the swim track of a randomly chosen individual over $1 \mathrm{~min}$. Although fish of ten produced wakes on the water's surface, we were still able to follow them and accurately determine their positions. Swim tracks were digitized at 5 min intervals during the first, third, fifth and seventh half-hours of the feeding period for each group. Swim speeds were converted to body lengths $\mathrm{s}^{-1}$ Swim path sinuosity was defined as the ratio of net to gross distance traveled. Hence, a high ratio indicates fish swimming in a relatively straight path, whereas a low ratio indicates a more convoluted swim path. Since fish were contained within an arena, they had to turn to avoid contacting the arena wall, and we felt that measuring sinuosity over long net distances could obfuscate differences between treatments. Therefore, sinuosity was calculated for short net distances equal to one-sixth the arena diameter. From the starting position, the net distance traveled was tallied until it equaled the one-sixth threshold, then sinuosity was calculated. From this new position the process was repeated, yielding multiple sinuosity measures for each digitized track. Preliminary observations suggested a one-sixth diameter would reduce arena artifacts, but still allow us to detect possible differences in swim path sinuosity between treatments. Sinuosity for each group/time period combination was calculated by averaging all sinuosity measurements for a given track and then averaging these for each time period. Two-dimensional analysis of swim tracks might be considered inadequate to characterize the movements of many fish, however, our observations indicated that both juvenile walleye pollock and sablefish foraged almost exclusively just below the water's surface, where food was dispensed. Therefore, we assumed that any errors in swim speed and swim path sinuosity measurements were relatively minor and comparable under both food distributions.

Statistical analysis. Due to differing levels of replication between walleye pollock and sablefish experiments, loss of data for the first week of the pollock experiment, and problems with heterogeneity of variance, we did not attempt to analyze our data using mul.ti-dimensional repeated measures ANOVA. Instead, we conducted comparisons between food distribution for each cómbination of species, week and hour of the feeding period. Although this approach is less efficient and less powerful than the multi-factor ANOVA approach, it was more practical given the limitations of our data. Data for swim speed, sinuosity, and growth showed no serious deviations from normality, as indicated by visual inspection of rankit plots (Sokal \& Rohlf 1969), and differences between food distributions were tested for significance with t-tests (Sokal \& 
Rohlf 1969). Where data were heteroscedastic, Satterthwaite's approximate degrees of freedom were utilized in determining significance (Day \& Quinn 1989). For congregation frequencies, there was often zero variance associated with the dispersed food treatments, and we therefore used Kruskal-Wallis ANOVA for all congregation analysis (Conover 1980). Because we had prior data and specific predictions regarding swim speed, differences between treatments for this variable were conducted with 1-tailed tests. Statistical tests for all other variables were 2 -tailed. Differences were considered significant at the $p<0.05$ level.

\section{RESULTS}

\section{Walleye pollock}

When not receiving food, juvenile walleye pollock cruised slowly about the arena. During the feeding period, fish moved about more rapidly, searching for food. After $1 \mathrm{wk}$, fish receiving the 2 food distributions had hegun to exhibit differences in search behavior. Fish receiving clumped food tended to congregate more than those receiving dispersed food, although this effect was not statistically significant (Fig. 1a, p = 0.061 for each hour). Fish fed clumped food swam faster than those fed dispersed food during the first $2 \mathrm{~h}$ of the $4 \mathrm{~h}$ feeding period (Fig. 2a, $\mathrm{p}=0.013,0.015$, 0.052 and 0.072 for Hours 1 to 4). Similarly, fish fed clumped food swam in a straighter path during the first $2 \mathrm{~h}$ of the feeding period (Fig. $3 \mathrm{a}, \mathrm{p}=0.037,0.036$, $0.056,0.056$ for Hours 1 to 4 ). Growth did not differ between food distributions after 1 wk, with fish receiving clumped and dispersed increasing their weights by $15.4 \%$ and $15.7 \%$, respectively (Fig. $4, \mathrm{p}=0.918$ ).

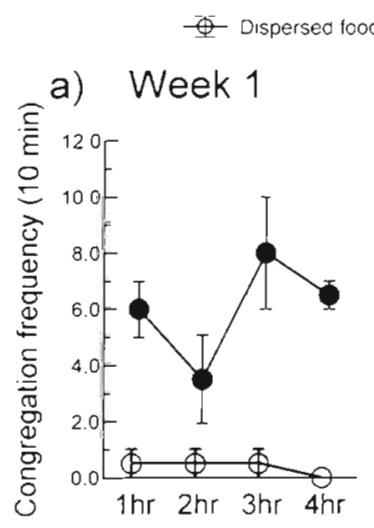

- Clumped food

b) Week 2

Fig. 1. Theragra chalcogramma. Mean number of congregations ( $\pm 1 \mathrm{SE}$ ) during $10 \mathrm{~min}$ focal durations for groups of juvenile walleye pollock receiving either dispersed or clumped food over a $4 \mathrm{~h}$ feeding period for (a) Week 1 and (b) Week 2
By the end of the second week, differences in search behavior had become more pronounced and consistent throughout the feeding period. Fish receiving clumped food congregated more frequently than those receiv-

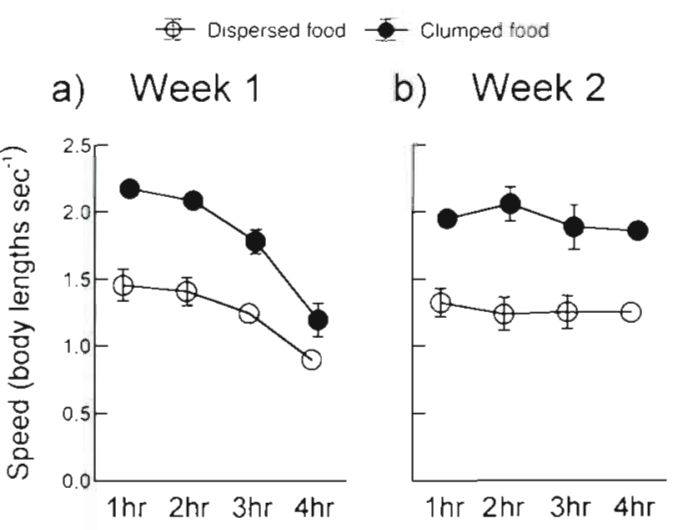

Fig. 2. Theragra chalcogramma. Mean swimming speed ( $\pm 1 \mathrm{SE})$ for juvenile walleye pollock receiving either dispersed or clumped food during a $4 \mathrm{~h}$ feeding period for (a) Week 1 and (b) Week 2

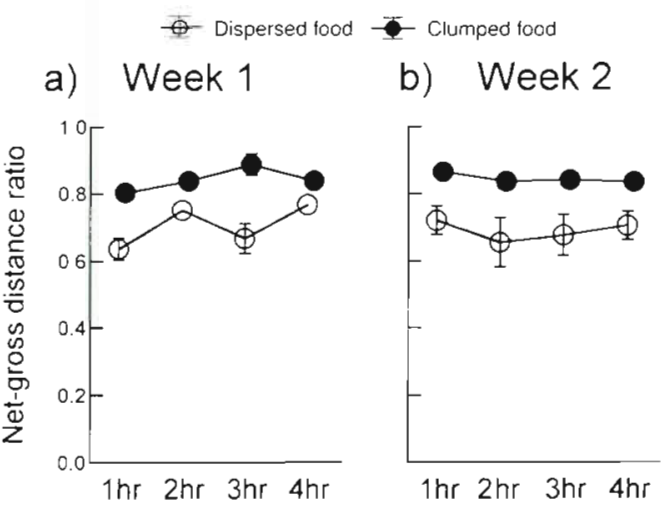

Fig. 3. Theragra chalcogramma. Mean swim path sinuosity, as measured by net-gross distance ratio ( $\pm 1 \mathrm{SE})$, for juvenile walleye pollock receiving either dispersed or clumped food during a $4 \mathrm{~h}$ feeding period for (a) Week 1 and (b) Week 2

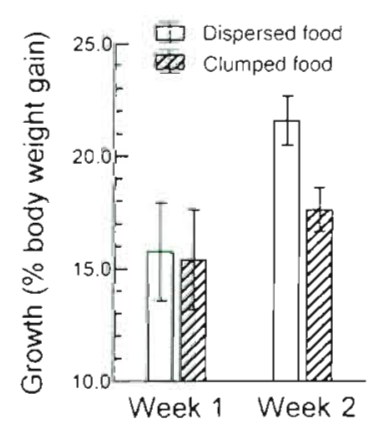

Fig. 4. Theragra chalcogramma. Mean growth ( $\pm 1 \mathrm{SE}$ ) for juvenile walleye pollock receiving either dispersed or clumped food for Weeks 1 and 2 
ing dispersed food throughout the feeding period (Fig. 1b, $p=0.013$ for each hour). Fish receiving clumped food also swam faster than those receiving dispersed food throughout the feeding period (Fig. $2 \mathrm{~b}$, $p=0.004,0.002,0.011$ and 0.001 for Hours 1 to 4 ) and swam in straighter paths during all but $1 \mathrm{~h}$ of the feeding period (Fig. 3b, $p=0.018,0.089,0.046$ and 0.039 for Hours 1 to 4 ). In contrast to Week 1, growth differed significantly between food distributions, with fish fed clumped food increasing their weight by $17.6 \%$, compared to $21.6 \%$ for fish fed dispersed food (Fig. 4, p = 0.034 ). In relative terms, fish fed clumped food experienced $19 \%$ lower growth than fish receiving dispersed food.

\section{Sablefish}

Like walleye pollock, juvenile sablefish cruised slowly about the arena when not being fed, but became more active when food was present. After $1 \mathrm{wk}$, sablefish receiving clumped food tended to congregate more frequently than those receiving dispersed food (Fig. 5a), but this was only significant during $2 \mathrm{~h}$ of the feeding period $(\mathrm{p}=0.085,0.007,0.016$ and 0.085 , for Hours 1 to 4 ). There were no differences in swimming speed between food distributions (Fig. 6a, p = 0.255, 0.088, 0.256 and 0.165 for Hours 1 to 4). Fish receiving clumped food swam in straighter paths, during the first $3 \mathrm{~h}$ of the feeding period, than did fish receiving dispersed food (Fig, $7 \mathrm{a}, \mathrm{p}=0.001$, $0.002,0.013$ and 0.068 , for Hours 1 to 4 ). For Week 1 , growth did not differ between food distributions ( $p=$ 0.870 ), with fish receiving clumped food increasing their weight by $45.8 \%$, compared to $45.0 \%$ for fish fed dispersed food (Fig. 8).

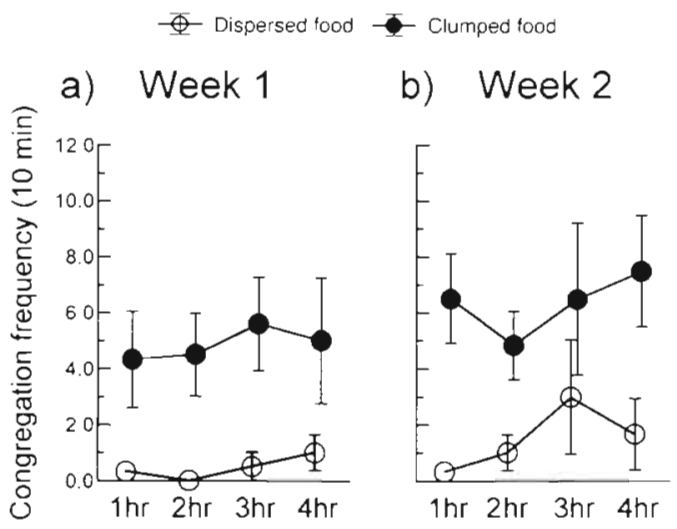

Fig. 5. Anaplopoma fimbria. Mean number of congregations ( $\pm 1 \mathrm{SE}$ ) during $10 \mathrm{~min}$ focal durations for groups of juvenile sablefish receiving either dispersed or clumped food during a $4 \mathrm{~h}$ feeding period for (a) Week 1 and (b) Week 2
By the end of the second week, differences between the 2 food distribution treatments, in terms of congregation frequency and swimming speed, were still inconsistent through the feeding period. Sablefish receiving clumped food congregated more frequently

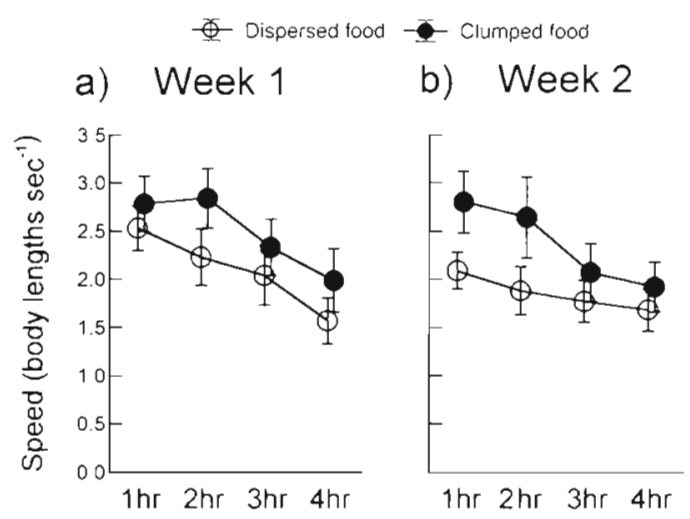

Fig. 6. Anaplopoma fimbria. Mean swimming speed ( $\pm 1 \mathrm{SE}$ ) for juvenile sablefish receiving either dispersed or clumped food during a $4 \mathrm{~h}$ feeding period for (a) Week 1 and (b) Week 2

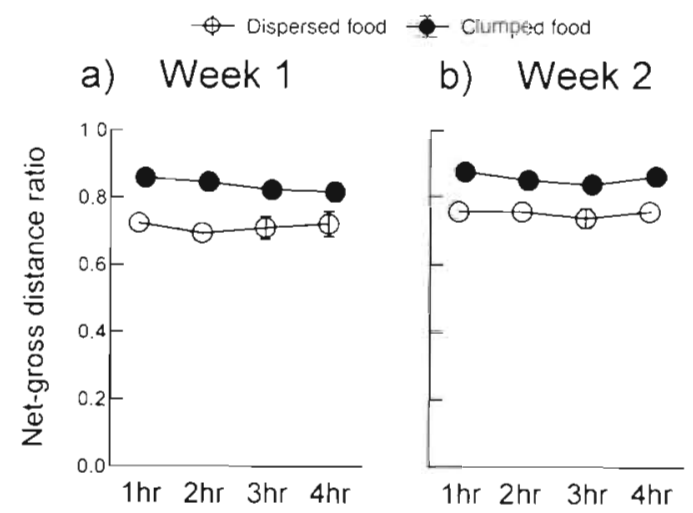

Fig. 7. Anaplopoma fimbria. Mean swim path sinuosity, as measured by net-gross distance ratio ( \pm 1 SE), for juvenile sablefish receiving either dispersed or clumped food during a 4 h feeding period for (a) Week 1 and (b) Week 2

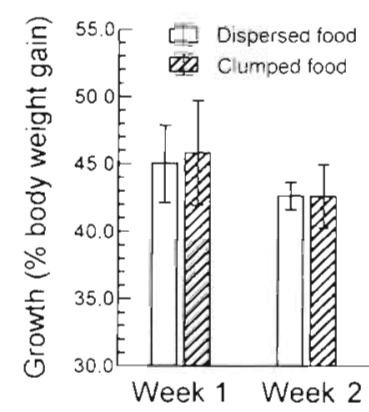

Fig. 8. Anaplopoma fimbria. Mean growth ( $\pm 1 \mathrm{SE}$ ) for juvenile sablefish receiving either dispersed or clumped food for Weeks 1 and 2 
than those receiving dispersed food only during the first half of the feeding period (Fig. 5b, $p=0.005,0.020$, 0.164 and 0.086 , for Hours 1 to 4 ), and swam faster during just the first hour (Fig. 6b, $p=0.044,0.075,0.220$ and 0.248 , for Hours 1 to 4 ). As was the case with pollock, differences in swim path sinuosity were more consistent throughout the feeding period, with sablefish receiving clumped food swimming straighter than those receiving dispersed food (Fig 7b, $p<0.001$ for Hours 1 and $4, p=0.017$ and 0.016 for Hours 2 and 3 ) Unlike walleye pollock, sablefish growth did not differ between food distributions for Week $2(p=0.992)$, with both increasing their weight by $42.6 \%$ (Fig. 8 ).

\section{DISCUSSION}

Prior research has demonstrated that juvenile walleye poilock utilize sucial foraging to locate and exploit clumped food, but forage more independently when food is dispersed (Ryer \& Olla 1992, 1995). This same behavior was observed in the current study, where juvenile wallcyc pollock, and to a lesser extent juvenile sablefish, congregated more frequently when food was clumped as opposed to dispersed. These congregations were not a result of fish aggregating at the site of a food clump, since no food was actually present at the moment congregations occurred. Rather, they were a manifestation of the greater social interaction and coordination of movement that occurs when fish forage socially. Associated with this greater social interaction was a change in the swimming speed of fish as they searched for food. After $2 \mathrm{wk}$ of acclimation, juvenile walleye pollock foraging for clumped food swam approximately $50 \%$ faster throughout the feeding period than fish foraging for dispersed food. Juvenile sablefish were less affected by food distribution, as individuals receiving clumped food swam faster than fish receiving dispersed food only during the first hour of foraging. Data for a variety of fish species indicate that energy consumption is nonlinearly related to swimming speed, such that it costs disproportionately more to swim faster (Tytler 1969, Puckett \& Dill 1984, Bernatchez \& Dodson 1985, Dabrowski 1986, Furnell 1987, Bushnell \& Brill 1991, Burggren \& Bemis 1992). As a result, we conclude that juvenile walleye pollock foraging for clumped food expended more energy than dispersed food foragers. This was confirmed by our measurement of fish weights; walleye pollock receiving clumped food experienced $19 \%$ lower growth than dispersed food foragers consuming the same quantity of food.

An increase in energy consumption associated with social foraging for clumped food will often be offset by proportionally greater increases in food consumption, since groups are more effective at locating prey that are spatially and temporally clumped in their distribution (Pitcher et al. 1982, Baird et al. 1991). As such, enhanced foraging opportunity may constitute one of the principle advantages of schooling (Pitcher et al. 1982, Baird et al. 1991, Ryer \& Olla 1992, 1995). However, the question remains: why do juvenile walleye pollock swim faster when foraging for clumped food than when foraging for dispersed food? Two possible explanations involve the relationship between optimal swimming speed and food detectability. In the first, clumps of food are probably more conspicuous than are individual food items, such that fish may be able to swim faster and still detect them (Gendron \& Staddon 1984). In this respect, clumping may be analogous to increased prey size or conspicuousness: white crappie Pomoxis annularis increase their search rate as prey increase in size (O'Brien et al. 1989), while European blackbirds Turdus merula search more rapidly when proy are more conspicuous in appearanre (Smith 1974). According to the second explanation, 'many eyes' may increase the probability that a given clump will be detected (Pitcher et al. 1982, Baird et al. 1991). This greater capability of groups to detect prey is comparable to the corporate vigilance effect, wherein grouping increases the probability that predators will be detected (Bertram 1978, Lazarus 1979). Under both of these scenarios, swimming faster allows more patches to be encountered and exploited. If this increase in food consumption exceeds the energetic costs of swimming faster, then the optimal swimming speed for clumped food should be greater than for dispersed food. Of course, within the confines of our arenas, it is unlikely that increased swimming speed would have been, strictly speaking, advantageous when foraging for clumped food. Whether they swam slow or fast, fish would still encounter all of the food clumps. However, we consider it unlikely that fish are able to distinguish between the energetic implications of clumped food in the field versus clumped food in the laboratory (Clark \& Mangel 1986). Therefore, rather than attempting to achieve optimality in foraging under our specific laboratory conditions, we consider it likely that fish were operating under a generalized rule of thumb (Krebs et al. 1983) 'swim faster when food is clumped', which would tend to approximate optimality under many natural conditions.

Alternatively, swimming faster when food is clumped may have nothing to do with optimality, but may instead result from intragroup competition. Individuals may accelerate their search rate, attempting to locate food before their school-mates, thereby gaining a greater share of any clumps they encounter. This has been referred to as the 'milkshake effect': imagine 2 people, each with their own straw, sharing a milkshake (Clark \& Mangel 1986). Under such conditions, swim- 
ming faster may actually be counterproductive, in terms of optimality, and fish may find themselves in a logical conundrum akin to the 'prisoner's dilemma' or 'tragedy of the commons' (Hardin 1968). In this scenario, the adopted behavior is inefficient from the perspective of optimality theory, but is nonetheless the evolutionarily stable strategy (Maynard Smith 1974).

Juvenile sablefish were not as responsive to our manipulation of food distribution. Although swimming speed was initially greater under clumped as opposed to dispersed food, this difference did not persist throughout the $4 \mathrm{~h}$ feeding period as it did for walleye pollock. Because we conducted few replicates of our food distribution treatments, this lack of statistical significant responsiveness, both in behavior and growth, may simply be an artifact of low statistical power. However, it is plausible that another factor, which was directly under our control, may have been responsible for our differing results with these 2 species: walleye pollock received $3 \%$ and sablefish received $6 \%$ daily rations. We had decided to give sablefish a larger ration based upon their rapid growth potential (Shenker \& Olla 1986) and our experience rearing them in the laboratory. This may have resulted in sablefish becoming partially satiated midway through the feeding period, possibly lessening differences in behavior between food distributions. Because ration size is confounded by species, no conclusion in this regard is possible; however, since ration size is known to mediate in other types of foraging behavior (Ryer \& Olla 1996), any future investigations of the linkage between food distribution, behavior and energetics would do well to consider ration size effects.

When searching for prey clumps, foragers often exhibit a ranging or 'extensive' mode of search, characterized by long movements in relatively straight lines (Bond 1980). Once prey are encountered, search mode changes to an 'intensive', or 'success-motivated search', pattern (Vinson 1977), characterized by shorter movements, frequent turning and reversal of direction. This change from extensive to intensive search has been observed for diverse taxa ranging from copepods (Williamson 1981) and insects (Bell 1985, Ferran et al. 1994) to larval fish (Hunter \& Thomas 1974, Coughlin et al. 1992) and birds (Smith 1974, Tome 1989). Extensive search allows foragers to cover large areas while searching for prey clumps. Once encountered, intensive search keeps the forager in the vicinity of the clump. As the clump becomes exhausted and encounter rates with prey decrease, transition back to extensive search occurs (Bell 1985). Although we did not quantify these different search phases in our experiments, this classification scheme characterizes what we observed in both juvenile walleye pollock and sablefish. For fish receiving clumped food, the intensive search phase which we characterized as milling behavior only lasted for several seconds at a time, with periods of minutes between intensive events. Hence our sinuosity measure, while slightly biased by the occasional inclusion of intensive events, should be considered as representative of extensive search. Classification of search behavior as either extensive or intensive may be less appropriate when food is dispersed, since fish interrupted their searching only long enough to intercept detected food pellets. Our observations would suggest that, rather than engaging in 2 separate search phases, both walleye pollock and sablefish foraging for dispersed food engaged in search behavior that was intermediate between the extensive and intensive search behavior observed in fish receiving clumped food.

The lower-sinuosity extensive search exhibited by fish receiving clumped food may have been attributable to the greater visibility of clumps, allowing fish to swim straighter and faster between turns and direction reversals. Social interactions may have also contributed to the lower search path sinuosity of clumped food foragers. Even in the loose groupings exhibited by facultative schoolers, individuals must coordinate their movements, giving up some degree of autonomy and independence of movement to maintain a minimum of group cohesion. For a school on the move, this should decrease the sinuosity of individual swim paths, assuming that the meanderings of the school occur at a spatial scale greater than that of the solitary individual. This straightening of the search path may also decrease energy consumption, since fewer turns and reversals are executed (Krohn \& Boisclair 1994); however, judging by the lower growth of fish receiving clumped food, any such savings were probably small compared to the added expense of swimming faster which accompanies clumped-food foraging.

In summary, the results of our study demonstrate that how food is distributed can affect foraging energetics. Juvenile walleye pollock receiving spatially and temporally clumped food swam faster and expended more energy in their foraging than fish receiving spatially and temporally dispersed food. This difference in energy expenditure was large enough in juvenile walleye pollock to produce significant differences in growth. For juvenile sablefish, differences in swimming speed were more modest and produced no significant differences in growth. Why these 2 facultative schoolers differed in this respect is unknown, but may be related to differences in the daily ration each species received. For both species, search paths were more sinuous among clumped food foragers. Both changes in swimming speed and search path sinuosity may have been the result of greater social interaction and coordination of movements between individuals in groups 
foraging for clumped food, as well as direct influences of food distribution. Increased swim speed in clumpedfood foragers may also represent an example of the competitively induced 'milkshake effect' hypothesized by Clark \& Mangel (1986), where fish adopt a search speed that is suboptimal, but evolutionarily stable.

Caution must be exercised in extrapolating the results of laboratory studies to field situations. For example, the spatial and temporal scales of our food distributions may differ from those commonly encountered in the field. However, if the behaviors we have observed are at all representative of those which occur in the field, we would predict that food distribution will be an important factor influencing the behavior and foraging energetics of some facultatively schooling fish species. In the pelagic environment, unlike in our laboratory experiments, expending more energy in the search for clumped food may actually increase growth under many siluations through increased encounters with prey. Another factor we did not address in this work is the influence of predation risk. It is well known that fish often modify their behavior when predators are present (see roviews by Dill 1987. Magurran et al. 1993) and recent work in our laboratory indicates that predation risk also influences the willingness of juvenile walleye pollock to expend greater effort in the search for clumped food (Ryer \& Olla unpubl.). Together, these studies suggest that a more complete understanding of the energetics and growth of fish must incorporate an appreciation of how fish behaviorally adapt to changes in their environment and the energetic consequences of these behavioral adaptations

\section{LITERATURE CITED}

Baird TA, Ryer CH, Olla BL (1991) Social enhancement of foraging on an ephemeral food source in juvenule walleye pollock, Theragra chalcogramma. Environ Biol Fishes 31: $307-311$

Bell. WJ (1985) Sources of information controllnng motor patterns in arthropod local search orientation. J Insect Physiol $31: 837-847$

Bernatchez L, Dodson JJ (1985) Influence of temperature and current speed on the capacity of lake whitefish (Coregonus clupeaformis) and cisco (C. artedii). Can J Fish Aquat Sci 42:1522-1529

Bertram BCR (1978) Living in groups: predators and prey In: Krebs JR, Davies NB (eds) Behavioural ecology, an evolutionary approach. Blackwell Scientific Publications, Oxford, p $64-96$

Boisciair D, Sirois P (1993) Testing assumptions of fish bioenergetics models by direct estimation of growth, consumption, and activity rates. Trans Am Fish Soc 122:784-796

Bond AB (1980) Optimal foraging in a uniform habitat: the search mechanism of the green lacewing. Anim Behav 28 $10-19$

Brodeur RD, Wilson MT (1996) Mesoscale acoustic patterns of juvenile walleye pollock (Theragra chalcogramma) in the western Gulf of Alaska. Can J Fish Aquat Sci 53:1951-1963
Brown JL (1964) The evolution of diversity in avian territorial systems. Wilson Bull 76:160-169

Burggren WW, Bemis WE (1992) Metabolism and ram gill ventilation in juvenile paddlefish, Polyodon spathula (Chondrostel: Polyodontidae). Physiol Zool 65:515-539

Bushnell PG, Brill RW (1991) Responses of swimming skipjack (Katsuwonus pelamis) and yellowfin (Thunnus albacares) tunas to acute hypoxia, and a model of their cardiorespiratory function. Physiol Zool 64:787-811

Clark CW, Mangel M (1986) The evolutionary advantages of group foraging. Theor Popul Biol 30:45-75

Conover WJ (1980) Practical nonparametric statistics. Wiley and Sons, New York

Coughlin DJ, Strickler JR, Sanderson B (1992) Swimming and search behaviour in clownfish. Amphiprion perideraion, larvae. Anim Behav 44:427-440

Dabrowski KR (1986) A new type of metabolism chamber for the determination of active and postprandial metabolism of fish, and consideration of results for coregonid and salmon juveniles. J Fish Biol 28:105-117

Day RW, Quinn GP (1989) Comparisons of treatments after an analysis of variance in ecology. Ecol Monogr 59:433-463

Dill LM (1987) Animal decision making and its ecologica! consequences: the future of aquatic ecology and behaviour. Can J Zool 65:803-811

Ferran A, Ettifouri M, Clement P, Bell WJ (1994) Sources of variability in the transition from extensive to intensive search in coccinellid predators (Homoptera Corcinellidae). J Insect Behav 7:633-647

Furnell DJ (1987) Partitioning of locomotor and feeding metabolism in sablefish (Anoplopoma fimbria). Can J Zool $65: 486-489$

Gendron RP, Staddon JER (1984) A laboratory simulation of foraging behavior: the effect of search rate on the probability of detecting prey. Am Nat 124:407-415

Grant JWA (1993) Whether or not to defend? The influence of resource distribution. Mar Behav Physiol 23:137-153

Grover JJ (1990) Feeding ecology of late-larval and earlyjuvenile walleye pollock Theragra chalcogramma from the Gulf of Alaska in 1987. Fish Bull 88:463-470

Grover JJ (1991) Trophic relationship of age-0 and age-1 walleye pollock Theragra chalcogramma collected together in the eastern Bering Sea. Fish Bull 89:719-722

Hamner WM (1984) Aspects of schooling in Euphausia superba. J Crustac Biol 1:67-74

Hamner WM, Strand SW, Gilmer RW (1983) Behavior of Antarctic krill, Euphausia superba: chemoreception, feeding, schooling, and molting. Science 220:433-435

Hardin G (1968) The tragedy of the commons. Science 162: $1243-1248$

Hart JL. (1973) Pacific fishes of Canada. Bull Fish Res Board Can 180:1-740

Hunter JR, Thomas GL (1974) Effect of prey distribution and density on the searching and feeding behaviour of larval anchovy Engraulis mordax Girard. In: Blaxter JHS (ed) The early life history of fish. Proc Int Symp Scottish Mar Biol Assoc, 1973. Springer-Verlag, New York, p 559-574

Krebs JR, Stephens DW, Sutherland WJ (1983) Perspectives in optimal foraging. In: Brush A.H, Clark GA (eds) Perspectives in ornithology. Cambridge Univ Press, Cambridge, p $165-221$

Krohn MM, Boisclair D (1994) Use of a stereo-video system to estimate the energy expenditure of free-swimming fish Cân J Fish Aquat Sci 51:1119-1127

Lazarus J (1979) The early warning function of flocking in birds: an experimental study with captive quelea. Anim Behav 27:855-865 
Mackas DL, Boyd CM (1979) Spectral analysis of zooplankton spatial heterogeneity. Science 204:62-64

Mackas DL, Denman KL, Abbott MR (1985) Plankton patchiness: biology in the physical vernacular. Bull Mar Sci 37: $652-674$

Magurran AE, Seghers BH, Carvalho GR, Shaw PW (1993) Evolution of adaptive variation in antipredator behaviour. Mar Behav Physiol 23:29-44

Maynard Smith J (1974) The theory of games and the evolution of animal conflicts. J Theor Biol 47:209-221

O'Brien DP (1989) Analysis of the internal arrangement of individuals within crustacean aggregations (Euphausiacea, Mysidacea). J Exp Mar Biol Ecol 128:1-30

O'Brien WJ, Evans BI, Browman HI (1989) Flexible search tactics and efficient foraging in saltatory searching animals. Oecologia 80:100-110

Pitcher TJ, Magurran AE, Winfield IJ (1982) Fish in larger shoals find food faster. Behav Ecol Sociobiol 10:149-151

Puckett KJ, Dill LM (1984) Cost of sustained and burst swimming to juvenile coho salmon (Oncorhynchus kisutch). Can J Fish Aquat Sci 41:1546-1551

Ryer CH, Olla BL (1992) Social mechanısms facilitating exploitation of spatially variable ephemeral food patches in a pelagic marine fish. Anim Behav 44:69-74

Ryer $\mathrm{CH}$, Olla BL (1995) Influences of food distribution on fish foraging behaviour. Anım Behav 49:411-418

Ryer $\mathrm{CH}$, Olla BL (1996) Growth depensation and aggression in laboratory reared coho salmon: the effect of food distribution and ration size. J Fish Biol 48:686-694

This article was submitted to the editor
Shenker JM, Olla BL (1986) Laboratory feeding and growth of juvenile sablefish. Anoplopoma fimbria. Can J Fish Aquat Sci 43:930-937

Smith JNM (1974) The food searching behaviour of two European thrushes. II: the adaptiveness of the search patterns. Behaviour 49:1-61

Sokal RR, Rohlf FJ (1969) Biometry. WH Freeman, San Francisco

Stavn RH (1971) The horizontal-vertical distribution hypothesis. Langmuir circulations and Daphnia distributions. Limnol Oceanogr 16:453-466

Tome MW (1989) Search-path characteristics of foraging ruddy ducks. Auk 106:42-48

Tytler P (1969) Relationship between oxygen consumption and swimming speed in the haddock, Melanogrammus aeglefinus. Nature 221:274-275

Vinson SB (1977) Behavioral chemicals in the augmentation of natural enemies. In: Ridgeway SBV (ed) Biological control by augmentation of natural enemies. Plenum, New York, p 237-279

Ware DM (1978) Bioenergetics of pelagic fish: theoretical change in swimming speed and ration with body size. J Fish Res Board Can 35:220-228

Williamson CE (1981) Foraging behavior of a freshwater copepod: frequency changes in looping behavior at high and low prey densities. Oecologia (Berl) 50:332-336

Zeldis JR, Jillett JB (1982) Aggregation of pelagic Munida gregaria (Fabricius) (Decapoda, Anomura) by coastal fronts and internal waves. J Plankton Res 4:839-857

Manuscript first received: February 4, 1997

Revised version accepted: April 30, 1997 